\title{
Descomposición de hojarasca y liberación de nutrientes en plantaciones de Acacia mangium (Mimosaceae) establecidas en suelos degradados de Colombia
}

\author{
Jeiner Castellanos-Barliza \& Juan Diego León Peláez \\ Universidad Nacional de Colombia, Sede Medellín, Facultad de Ciencias Agropecuarias, Medellín, Colombia, Calle \\ 59A 63-20, 14-330; jeinercast@gmail.com, jdleon@unal.edu.co
}

\author{
Recibido 20-I-2010. Corregido 05-VIII-2010. Aceptado 03-IX-2010.
}

\begin{abstract}
Litter decomposition and nutrient release in Acacia mangium plantations established on degraded soils of Colombia. Several factors control the decomposition in terrestrial ecosystems such as humidity, temperature, quality of litter and microbial activity. We investigated the effects of rainfall and soil plowing prior to the establishment of Acacia mangium plantations, using the litterbag technique, during a six month period, in forests plantations in Bajo Cauca region, Colombia. The annual decomposition constants $(k)$ of simple exponential model, oscillated between 1.24 and 1.80, meanwhile $k_{1}$ y $k_{2}$ decomposition constants of double exponential model were $0.88-1.81$ and $0.58-7.01$. At the end of the study, the mean residual dry matter (RDM) was $47 \%$ of the initial value for the three sites. We found a slow $\mathrm{N}$, Ca and $\mathrm{Mg}$ release pattern from the A. mangium leaf litter, meanwhile, phosphorus $(\mathrm{P})$ showed a dominant immobilization phase, suggesting its low availability in soils. Chemical leaf litter quality parameters (e.g. N and $\mathrm{P}$ concentrations, $\mathrm{C} / \mathrm{N}, \mathrm{N} / \mathrm{P}$ ratios and phenols content) showed an important influence on decomposition rates. The results of this study indicated that rainfall plays an important role on the decomposition process, but not soil plowing. Rev. Biol. Trop. 59 (1): 113-128. Epub 2011 March 01.
\end{abstract}

Key words: Acacia mangium, soil plowing, litter bags, nutrient release, decomposition rates, soil reclamation.

La capa de hojarasca produce un abrigo orgánico sobre la superficie de los suelos, dando por resultado un microclima edáfico peculiar, y condiciones adecuadas para un espectro más amplio de organismos. Su descomposición contribuye a la regulación del ciclo de nutrientes y de la productividad primaria, así como al mantenimiento de la fertilidad del suelo forestal (Wang et al. 2008). Como proceso, la descomposición es clave para el funcionamiento de los bosques, ya que si los nutrientes son liberados rápidamente, pueden perderse por lixiviación edáfica o por volatilización (Palma et al. 1998, Schlesinger 2000). Por el contrario, si la descomposición es muy lenta, el capital de nutrientes disponible para las plantas puede ser insuficiente, y limitar el crecimiento y desarrollo (Jordan 1985, Swift \& Anderson 1989, Bubb et al. 1998, Montagnini \& Jordan 2002).

El patrón general para la pérdida de peso de la hojarasca en descomposición comprende dos fases de estado, una inicial de rápido desarrollo por el lavado de compuestos solubles y la descomposición de materiales lábiles (e.g. azúcares, algunos fenoles, almidones y proteínas), y una segunda más lenta, como resultado de la lenta descomposición de elementos recalcitrantes como celulosa, hemicelulosa, taninos y lignina (Arellano et al. 2004, GomaTchimbakala \& Bernhard-Reversat 2006). Por otra parte, durante la descomposición de la materia orgánica pueden llegar a diferenciarse tres fases para la liberación de nutrientes: una 
inicial de rápida liberación de componentes solubles, en la que dominan los procesos de lavado, seguida por una fase de inmovilización, y finalmente una fase de liberación neta (Weerakkody \& Parkinson 2006a). Esta liberación puede tomar varios caminos dependiendo de diferentes factores como la humedad, la temperatura, la disponibilidad de nutrientes en el suelo, la especie, la edad y, fundamentalmente, la calidad de la hojarasca (concentraciones de $\mathrm{N}$ y $\mathrm{P}$; relaciones $\mathrm{C} / \mathrm{N}$ y $\mathrm{N} / \mathrm{P}$; contenido de lignina, taninos, etc.). Estas características de calidad de la hojarasca pueden determinar a su vez la biomasa microbiana y la mineralización de los nutrientes (Attiwill \& Adams 1993, Bubb et al. 1998, McGrath et al. 2000, Kumar \& Agrawal 2001, Villela \& Proctor 2002, Singh et al. 2004, Weerakkody \& Parkinson 2006 b, Liao et al. 2006, Huang et al. 2007).

La descomposición y liberación de nutrientes de la hojarasca, constituyen procesos clave para garantizar el adecuado funcionamiento de los ciclos biogeoquímicos, y con ellos favorecer unas apropiadas condiciones físicas, químicas y biológicas de los suelos. En el caso de la recuperación de áreas degradadas por actividades de minería superficial, la restitución de estos procesos es fundamental, ya que la vegetación ha sido eliminada y con ella el funcionamiento de estos ciclos (León et al. 2008).

La especie Acacia mangium además de ser una de las más utilizadas en sistemas agroforestales en los trópicos cálidos y húmedos, ha sido ampliamente usada en programas de restauración de suelos afectados por la minería (Tsukamoto \& Sabang 2005, Torres \& del Valle 2007). Esto obedece, entre otras razones, a su rápido crecimiento, alta productividad y tolerancia a condiciones desfavorables de diferentes tipos de suelos (Torres \& del Valle 2007), y por la efectividad que muestra en el establecimiento de asociaciones simbióticas con microorganismos del suelo (e.g. hongos micorrícicos, bacterias fijadoras de $\mathrm{N}_{2}$ atmosférico; Lim 1988, Duponnois \& Ba 1999). En la década de los noventa se establecieron en Colombia plantaciones de la especie en los departamentos de Córdoba y Antioquia, principalmente con fines comerciales y de restauración (Torres \& del Valle 2007). En la región del Bajo Cauca Antioqueño, las plantaciones establecidas con esta especie tuvieron esencialmente objetivos de recuperación de suelos degradados por minería aurífera, y de restauración ecológica; no obstante el conocimiento que actualmente se tiene de su funcionamiento es mínimo y queda aún por determinarse su efectividad para tales fines, de tal manera que pueda seguir siendo empleada en otras áreas degradadas del país.

El objetivo de este estudio fue describir los procesos de descomposición y liberación de nutrientes de la hojarasca foliar en plantaciones de Acacia mangium, establecidas sobre suelos degradados por minería de oro en el Bajo Cauca Antioqueño. Para ello, se evaluaron como factores de potencial afectación sobre los procesos estudiados, el régimen pluviométrico, la práctica de subsolado del suelo realizada previo establecimiento de las plantaciones, y algunos parámetros de calidad de la hojarasca foliar.

\section{MATERIALES Y MÉTODOS}

Área de estudio: El estudio se realizó entre abril y septiembre del 2007, en la región del Bajo Cauca Antioqueño, en las últimas estribaciones de la Cordillera Central en los sitios conocidos como Río Rayo, municipio de Tarazá $\left(07^{\circ} 31^{\prime} 43.6^{\prime \prime}\right.$ N.- $75^{\circ} 21^{\prime} 04^{\prime \prime}$ W) y Jardín, municipio de Cáceres (07\%4'30.2" $\left.\mathrm{N}-75^{\circ} 14^{\prime} 26.2^{\prime \prime} \mathrm{W}\right)$, al nororiente de Antioquia, Colombia. En el municipio de Tarazá la temperatura media anual es de $27^{\circ} \mathrm{C}$ y la precipitación media anual de $3133 \mathrm{~mm}$. En el municipio de Cáceres la temperatura media anual es de $28^{\circ} \mathrm{C}$ y la precipitación media anual de $2771 \mathrm{~mm}$ (IGAC 2007).

Las plantaciones de Acacia mangium estudiadas tenían una edad de 10-11 años y fueron establecidas por la Corporación Autónoma Regional del Centro de Antioquia (CORANTIOQUIA) para rehabilitar áreas degradadas por procesos ancestrales de extracción del oro, que ocasionaron la destrucción de todos los horizontes del suelo y la vegetación. En 
estos suelos se verifica la pérdida de materia orgánica de sus primeros horizontes, así como buena parte del banco natural de semillas y del componente microbiológico del suelo (Osorio 2000). Además, el material del suelo que queda sobre la superficie exhibe serias limitaciones físicas y químicas para un adecuado crecimiento vegetal, destacándose el daño de la estructura del suelo y la contaminación por mercurio, con lo cual, el restablecimiento exitoso de actividades agrícolas y pecuarias es bastante restringido (León et al. 2008). Dada la alta compactación de estos suelos, algunos lotes fueron arados superficialmente con tractor (tratamiento de subsolado) para mejorar sus condiciones físicas. Los rodales tenían un área basal entre 24.3 y $30.1 \mathrm{~m}^{2} /$ ha y un volumen de $185-200 \mathrm{~m}^{3} / \mathrm{ha}$. Los suelos se caracterizaron por su alta acidez, bajo contenido de materia orgánica y macronutrientes y alto contenido de Fe (Cuadro 1). La precipitación total durante el período de estudio fue $2394.54 \mathrm{~mm}$ en Jardín y 3 212.29mm en Río Rayo (Fig. 1).
Métodos de campo: Se empleó la técnica de bolsas de descomposición, construidas con malla plástica de $2 \mathrm{~mm}$ de poro, para evitar pérdidas de material por la fragmentación, y la exclusión de algunos descomponedores importantes (Wieder \& Lang 1982, Sundarapandian \& Swamy 1999). El tamaño de las bolsas fue de $20 \times 20 \mathrm{~cm}$, cada una de las cuales se llenó con $10 \mathrm{~g}$ de hojas recién caídas desde el dosel, secadas a $65^{\circ} \mathrm{C}$. En cada uno de los sitios de estudio se colocaron 18 bolsas, las cuales se fijaron en grupos de seis bolsas alrededor de la base de tres árboles por sitio, para un total de 54 bolsas en los tres sitios. Esta distribución se adoptó con el objetivo de evaluar la influencia del tratamiento del subsolado y de la precipitación, sobre el proceso de descomposición. Para evaluar el primer factor, se establecieron sitios bajo la misma condiciones climáticas pero con diferente pre-tratamiento del suelo (JS: Jardín subsolado y JNS: Jardín no subsolado). Para evaluar la incidencia de la lluvia, se colocaron las bolsas en sitios con registro pluviométrico

CUADRO 1

Características de las plantaciones de A. mangium y de los suelos en los sitios estudiados en el Bajo Cauca, Colombia

TABLE 1

A. mangium stands characteristics and soil properties of the study sites in the Bajo Cauca region, Colombia

\begin{tabular}{|c|c|c|c|c|c|c|c|c|c|c|c|c|c|c|}
\hline \multicolumn{15}{|c|}{ Características estructurales de los rodales } \\
\hline \multicolumn{2}{|c|}{ Sitios } & \multicolumn{3}{|c|}{$\begin{array}{c}\mathrm{N} \\
\text { (Individuos/ha) }\end{array}$} & \multicolumn{2}{|c|}{$\begin{array}{l}\text { DAP } \\
(\mathrm{cm})\end{array}$} & \multicolumn{2}{|c|}{$\begin{array}{c}\mathrm{G} \\
\left(\mathrm{m}^{2} / \mathrm{ha}\right)\end{array}$} & \multicolumn{2}{|c|}{$\begin{array}{c}\mathrm{V} \\
\left(\mathrm{m}^{3} / \mathrm{ha}\right)\end{array}$} & \multicolumn{2}{|c|}{$\begin{array}{c}\text { B } \\
\text { (ton/ha) }\end{array}$} & \multicolumn{2}{|c|}{$\begin{array}{c}\mathrm{H} \\
(\mathrm{m})\end{array}$} \\
\hline JS & & \multicolumn{3}{|c|}{1920} & \multicolumn{2}{|c|}{16.5} & \multicolumn{2}{|c|}{45.5} & \multicolumn{2}{|c|}{321} & \multicolumn{2}{|c|}{268.8} & \multicolumn{2}{|c|}{20.1} \\
\hline JNS & & \multicolumn{3}{|c|}{640} & \multicolumn{2}{|c|}{21.7} & \multicolumn{2}{|c|}{25.5} & \multicolumn{2}{|c|}{192.7} & \multicolumn{2}{|c|}{167.9} & \multicolumn{2}{|c|}{22.0} \\
\hline RS & & \multicolumn{3}{|c|}{1680} & & .0 & \multicolumn{2}{|c|}{53.0} & \multicolumn{2}{|c|}{314.4} & \multicolumn{2}{|c|}{293.9} & \multicolumn{2}{|c|}{17.2} \\
\hline \multicolumn{15}{|c|}{ Características químicas de los suelos } \\
\hline \multirow{2}{*}{ Sitios } & \multirow{2}{*}{$\mathrm{pH}$} & MO & $\mathrm{N}$ & $\mathrm{C}$ & \multirow{2}{*}{$\mathrm{C} / \mathrm{N}$} & $\mathrm{Ca}$ & $\mathrm{Mg}$ & $\mathrm{K}$ & $\mathrm{Al}$ & $\mathrm{Cu}$ & $\mathrm{Fe}$ & $\mathrm{Mn}$ & $\mathrm{Zn}$ & $\mathrm{P}$ \\
\hline & & & $\%$ & & & \multicolumn{3}{|c|}{$\mathrm{cmol}(+) / \mathrm{kg}$} & \multicolumn{6}{|c|}{$\mathrm{mg} / \mathrm{kg}$} \\
\hline JS & 4.6 & 15.76 & 0.64 & 9.14 & 14.28 & 3.28 & 3.17 & 0.79 & 0.7 & 1.7 & 49.4 & 41.9 & 5.1 & 9.10 \\
\hline JNS & 4.1 & 28.59 & 0.56 & 16.58 & 29.61 & 4.36 & 2.55 & 0.75 & 0.8 & 0.9 & 27.6 & 41.2 & 2.3 & 3.99 \\
\hline RS & 4.1 & 7.09 & 0.43 & 4.11 & 9.56 & 4.28 & 3.24 & 0.31 & 0.6 & 2.8 & 323.0 & 130.0 & 4.0 & 12.20 \\
\hline $\mathrm{SC}$ & 5.4 & 6.14 & 0.24 & 3.56 & 14.83 & 2.80 & 2.53 & 0.21 & - & 3.9 & 23.6 & 22.9 & 2.4 & 2.48 \\
\hline
\end{tabular}

N: Densidad, DAP: diámetro medido a la altura del pecho $(1.3 \mathrm{~m}), \mathbf{G}$ : área basal, V: volumen de madera, B: biomasa, H: altura de los individuos. pH: determinado en solución suelo-agua 1:2. Sitios experimentales: JS: Jardín Subsolado, JNS: Jardín No Subsolado, RS: Río Rayo Subsolado, SC: Sitio Control (UNAL-CORANTIOQUIA 2008).N: Stand density, DAP: diameter at breast height $(1.3 \mathrm{~m}), \mathbf{G}$ : Stand basal area, V: stand wood volume, B: stand biomass, H: mean canopy height. pH: determined into soil-water mixture (1:2). Experimental sites: JS: Jardín site, plowed soil, JNS: Jardín site, nonplowed soil, RS: Río Rayo site, plowed soil, SC: Control site (UNAL-CORANTIOQUIA 2008). 


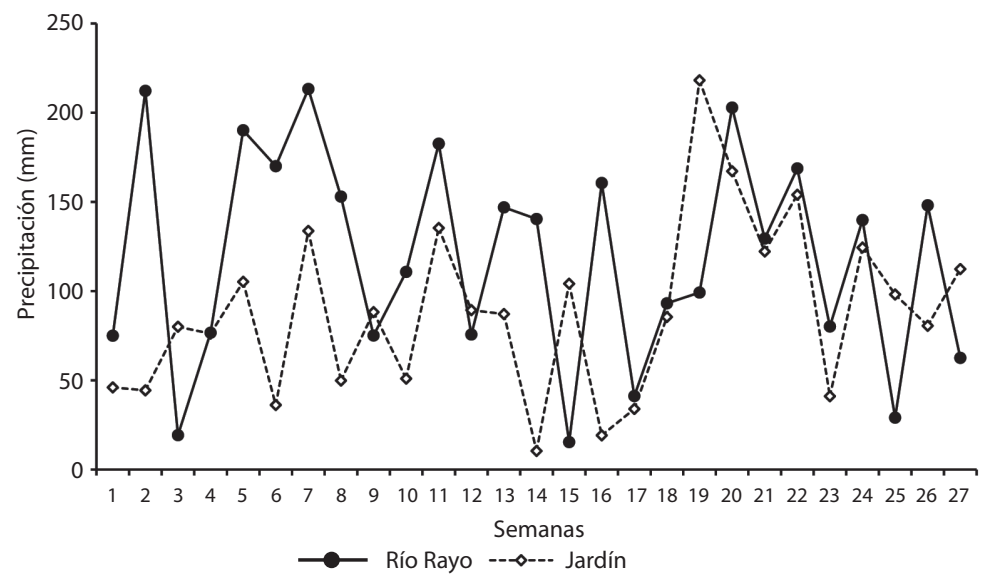

Fig. 1. Precipitación semanal durante el período de estudio en las plantaciones de A. mangium del Bajo Cauca, Colombia.

Fig. 1. Weekly rainfall during the study period in the A. mangium plantations in the Bajo Cauca region, Colombia.

diferente (RS: Río Rayo subsolado y JS: Jardín subsolado). Mensualmente se retiraron tres bolsas por sitio, para un total de nueve bolsas por evento de muestreo. Adicionalmente, de cada sitio fueron tomadas muestras del suelo superficial para análisis químico. Para el registro de la precipitación se instalaron cinco pluviómetros alrededor de las plantaciones, los cuales se midieron semanalmente.

Métodos de laboratorio: Las bolsas retiradas de campo fueron conducidas al laboratorio, en donde el material fue extraído y limpiado manualmente con un pincel para eliminar raíces, pequeños invertebrados, y partículas de suelo. Posteriormente el material fue secado a $65^{\circ} \mathrm{C}$ hasta obtener peso constante. Se determinaron las concentraciones de carbono (C), por combustión seca a través de un analizador LECO CNS-2000; N por micro-Kjeldahl, $\mathrm{P}$ por digestión de ácidos y espectrofotometría ultravioleta visible. Los cationes $\mathrm{K}, \mathrm{Ca}$ y $\mathrm{Mg}$ por digestión vía seca y absorción atómica. Las muestras de suelo fueron secadas al aire, tamizadas en malla $2.0 \mathrm{~mm}$ y preparadas para análisis de fertilidad. La lignina se determinó por el método de detergentes ácidos y del $\mathrm{KMnO}_{4}$
(Horwitz 2003, Tejada 1992). Los fenoles fueron extraídos con metanol durante cinco días y analizados por el método colorimétrico de Folin-Ciocalteu (Singleton \& Rossi 1965). Los resultados fueron expresados como mg de ácido gálico/100g de extracto.

Procesamiento y análisis de datos: $\mathrm{La}$ evolución de la materia seca residual (MSR) y los patrones de liberación de nutrientes se compararon entre sitios mediante análisis de varianza de una vía, previa comprobación de la normalidad y homocedasticidad de los datos. Cuando las varianzas fueron significativas, las diferencias se determinaron mediante la prueba de Tukey. Las cantidades residuales de los nutrientes en cada momento de tiempo, fueron calculadas mediante el producto de la MSR por la concentración en ese momento, dividido por la concentración inicial. Para determinar la asociación entre la MSR y algunos factores como la precipitación total, el tratamiento de subsolado y los parámetros de calidad del sustrato, fueron calculados coeficientes de correlación de Pearson. Para describir la evolución de la MSR, se ajustaron mediante regresión no lineal dos modelos de descomposición. El primero 
fue el simple exponencial negativo (Olson 1963), que describe la Ecuación 1.

$$
X t / X o=e^{-k t}
$$

Donde: $X t$ : peso seco del material remanente en un tiempo $t(\mathrm{~g}), X o$ : peso seco inicial $(\mathrm{g}), k$ : Constante de descomposición, $t$ : tiempo transcurrido (días). A partir de esta constante, se calculó la vida media o el tiempo necesario para alcanzar una descomposición de la hojarasca del 50\% $\left(\mathrm{t}_{0.5}\right)$ y del $99 \%\left(\mathrm{t}_{0.99}\right): t_{0.5}=-0.693 / k$ y $t_{0.99}=-4.605 / k($ Olson 1963, Arunachalam \& Singh 2002).

El segundo modelo fue el doble exponencial negativo (Bunnell \& Tait 1974), que tiene en cuenta las fracciones de fácil y difícil descomposición (Ecuación 2).

$$
X t / X_{0}=p e^{-k l t}+(1-p) e^{-k 2 t}
$$

Donde, $X_{0}=$ cantidad inicial de material $(\mathrm{g})$, $X_{t}=$ cantidad de material remanente tras un tiempo $t(\mathrm{~g}), p=$ parámetro de la fracción lábil,
( $1-p)=$ parámetro de la fracción recalcitrante, $k_{f}=$ constante de descomposición de la fracción lábil, $k_{2}=$ constante de descomposición de la fracción recalcitrante, $t=$ tiempo que transcurre entre $X_{0}$ y $X_{t}$. Para la selección de los modelos se emplearon como indicadores de ajuste, el coeficiente de determinación $\left(\mathrm{R}^{2}\right)$, el estadístico Durbin-Watson (D-W) y la suma de cuadrados del error. Los procesamientos estadísticos fueron realizados en el programa Statgraphics Centurion XV (StatPoint Technologies, Inc).

\section{RESULTADOS}

Evolución de la materia seca residual: Las tendencias en la pérdida de peso durante el estudio fueron similares entre sitios (Tukey $\mathrm{p}>0.05$ ) (Fig. 2). Con respecto al material inicialmente colocado en las bolsas de descomposición, la MSR representó al finalizar el experimento, $55 \%$ en JS, $41 \%$ en JNS y $42 \%$ en RS.

Velocidad de descomposición de la hojarasca foliar: Los modelos evaluados

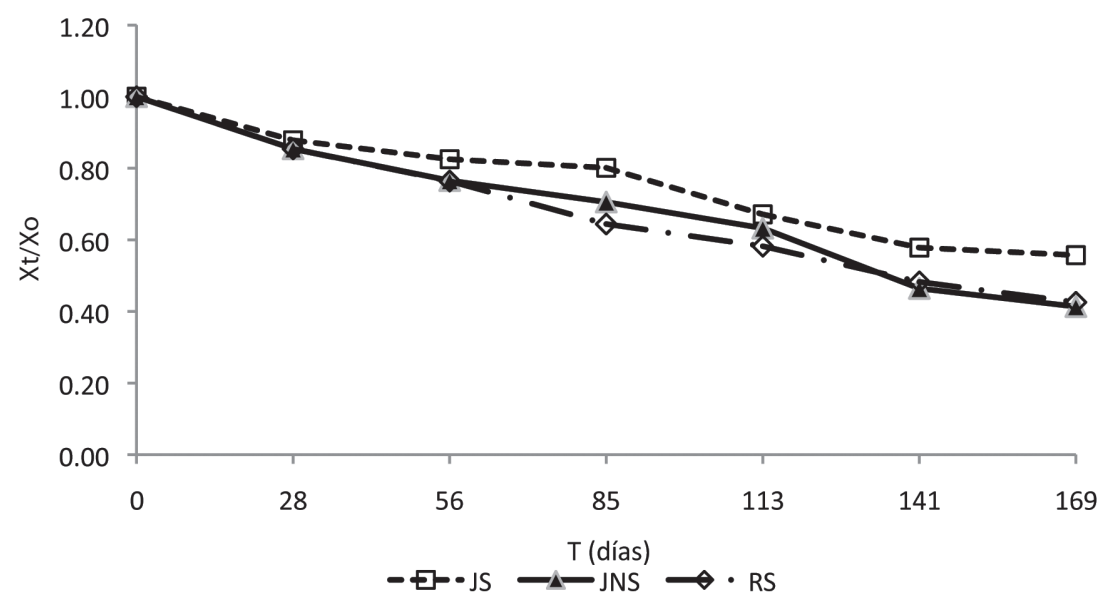

Fig. 2. Materia seca residual $\left(\mathrm{X}_{\mathrm{t}} / \mathrm{X}_{\mathrm{o}}\right)$ de la hojarasca foliar en tres sitios localizados en las plantaciones de Acacia mangium del Bajo Cauca, Colombia. Sitios experimentales: JS: Jardín subsolado, JNS: Jardín no subsolado, RS: Río Rayo subsolado.

Fig. 2. Ash-free residual dry matter $\left(\mathrm{X}_{\mathrm{t}} / \mathrm{X}_{\mathrm{o}}\right)$ from leaf litter for A. mangium plantations in three sites located in the Bajo Cauca region, Colombia. Experimental sites: JS: Jardín site, plowed soil, JNS: Jardín site, non-plowed soil, RS: Río Rayo site, plowed soil. 
mostraron buenos ajustes (Cuadro 2), siendo el mejor el doble exponencial, con constantes $k_{1}$ y $k_{2}$ similares entre JS y RS; en contraste, para JNS la constante $k_{2}$ fue considerablemente alta con respecto a la $k_{1}$. Para RS se obtuvieron los menores tiempos de residencia de la hojarasca foliar $\left(\mathrm{t}_{0.5}=4.6\right.$ meses y $t_{0.99}=2.6$ años). Las tasas de descomposición del modelo simple exponencial siguieron el modelo decreciente $\operatorname{RS}(k=1.80)>\operatorname{JNS}(k=1.72)>\mathrm{JS}(k=1.24)$.

Liberación de nutrientes durante la descomposición de la hojarasca foliar: Los nutrientes de mayor y menor abundancia en la MSR fueron, respectivamente, N y P. Para ambos nutrientes se presentó en los tres sitios un patrón dominante de incremento en la concentración. Así, al final del estudio, sus concentraciones aumentaron entre 25 y $52 \%$ para N y entre 150 y $325 \%$ para $\mathrm{P}$ (Cuadro 3).

La liberación de $\mathrm{N}$ fue lenta en los tres sitios hasta el día 113 (4.7 meses) y mayor hacia el final del estudio (Fig. 3). El Ca y el Mg mostraron una tendencia similar a la del $\mathrm{N}$, más clara para JNS y RS. Para el P se encontraron como procesos dominantes la inmovilización e incrementos en la MSR, siendo las ganancias al final del experimento (día 169) superiores incluso al $150 \%$ en JNS y RS. A diferencia de JNS, en JS y RS la liberación de K se presentó a lo largo de todo el estudio, siendo en general, similar a la de $\mathrm{Mg}$.

Evolución de la calidad de la hojarasca foliar: Los parámetros $\mathrm{C} / \mathrm{N}, \mathrm{N} / \mathrm{P}$ y fenoles totales mostraron fuerte disminución entre el inicio

CUADRO 2

Modelos de regresión ajustados para la materia seca residual en función del tiempo para la hojarasca foliar de A. mangium en el Bajo Cauca, Colombia

TABLE 2

Fitted regression models for residual dry matter as a function of time for A. mangium leaf litter in the Bajo Cauca region, Colombia

\begin{tabular}{|c|c|c|c|c|c|c|c|c|c|c|}
\hline \multicolumn{11}{|c|}{ Modelo 1} \\
\hline Sitio & Modelo & $\mathrm{t}_{0.5}(\mathrm{años})$ & $\mathrm{t}_{0.99}(\mathrm{a} n ̃ o s)$ & $\mathrm{k}(1 / \mathrm{año})$ & \multicolumn{2}{|c|}{$\mathrm{R}^{2}(\%)$} & \multicolumn{2}{|c|}{ SCE } & \multicolumn{2}{|c|}{ D-W } \\
\hline JS & $10 * \mathrm{e}^{(-0.0034127 * \mathrm{t})}$ & 0.56 & 3.70 & 1.25 & \multicolumn{2}{|c|}{89.44} & \multicolumn{2}{|c|}{3.95} & \multicolumn{2}{|c|}{2.19} \\
\hline JNS & $10 * \mathrm{e}^{\left(-0.00471566^{*} \mathrm{t}\right)}$ & 0.40 & 2.70 & 1.72 & \multicolumn{2}{|c|}{86.82} & \multicolumn{2}{|c|}{8.29} & \multicolumn{2}{|c|}{1.07} \\
\hline RS & $10 * \mathrm{e}^{(-0.00493899 * \mathrm{t})}$ & 0.38 & 2.60 & 1.80 & \multicolumn{2}{|c|}{96.20} & & 10 & \multicolumn{2}{|c|}{2.71} \\
\hline \multicolumn{11}{|c|}{ Modelo 2} \\
\hline Sitio & \multicolumn{4}{|c|}{ Modelo } & $\mathrm{p}$ & $\mathrm{k}_{1}$ & $\mathrm{k}_{2}$ & $\mathrm{R}^{2}$ & SCE & $\mathrm{D}-\mathrm{W}$ \\
\hline JS & \multicolumn{4}{|c|}{$1.1885 * \mathrm{e}^{(-0.88164 * \mathrm{t})}-0.1885 * \mathrm{e}^{(-0.58144 * \mathrm{t})}$} & 1.18 & 0.88 & 0.58 & 89.49 & 0.03 & 2.23 \\
\hline JNS & \multicolumn{4}{|c|}{$1.0035 * \mathrm{e}^{(-1.52628 * \mathrm{t})}-0.0035 * \mathrm{e}^{(-7.01709 * \mathrm{t})}$} & 1.00 & 1.52 & 7.01 & 87.90 & 0.07 & 1.20 \\
\hline RS & \multicolumn{4}{|c|}{$1.0039 * \mathrm{e}^{(-1.81023 * \mathrm{t})}-0.0039 * \mathrm{e}^{(-1.72762 * \mathrm{t})}$} & 1.00 & 1.81 & 1.72 & 96.18 & 0.02 & 2.68 \\
\hline
\end{tabular}

Xo: peso seco inicial $(10 \mathrm{~g}), \boldsymbol{k}$ : tasa de descomposición anualizada, $\boldsymbol{k}_{\mathbf{1}}$ : constante de descomposición anualizada de la fracción lábil, $\boldsymbol{k}_{2}$ : constante de descomposición anualizada de la fracción recalcitrante, $\boldsymbol{p}$ : proporción de compuestos lábiles, (1-p): proporción de compuestos recalcitrantes, $\mathbf{R}^{\mathbf{2}}$ : coeficiente de determinación, SCE: suma de cuadrados del error, $\mathbf{D}-\mathbf{W}$ : estadístico Durbin-Watson, $\mathbf{t}_{\mathbf{0 . 5}}=$ tiempo necesario para alcanzar una descomposición del $50 \%$ de la hojarasca, $\mathbf{t}_{\mathbf{0 . 9 9}}=$ tiempo necesario para alcanzar una descomposición del $99 \%$ de la hojarasca. Sitios experimentales: JS: Jardín subsolado, JNS: Jardín no subsolado, RS: Río Rayo subsolado.

Xo: original mass $(10 \mathrm{~g}), \boldsymbol{k}$ : yearly decomposition rate, $\boldsymbol{k}_{\mathbf{1}}$ : yearly decomposition rate for labile fraction, $\boldsymbol{k}_{2}$ : yearly decomposition rate for recalcitrant fraction, $p$ : proportion of labile compounds, $(\mathbf{1}-\boldsymbol{p})$ : proportion of recalcitrant compounds, $\mathbf{R}^{\mathbf{2}}$ : determination coefficient, $\mathbf{S C E}$ : sum of squared errors, D-W: Durbin-Watson statistics, $\mathbf{t}_{\mathbf{0} . \mathbf{5}}=$ decomposition time for half of the leaf litter, $\mathbf{t}_{\mathbf{0 . 9 9}}=$ decomposition time for $99 \%$ of the leaf litter. Experimental sites: JS: Jardín site, plowed soil, JNS: Jardín site, non-plowed soil, RS: Río Rayo site, plowed soil. 


\section{CUADRO 3}

Dinámica de la concentración de nutrientes en la MSR de la hojarasca foliar en las plantaciones de A. mangium en los tres sitios estudiados en el Bajo Cauca, Colombia. Valores expresados en \%

TABLE 3

Dynamics of leaf litter nutrient concentrations in residual dry mater of A. mangium plantations in the three sites studied in the Bajo Cauca region, Colombia. Values expressed in \%

\begin{tabular}{|c|c|c|c|c|c|c|c|c|c|c|c|c|c|c|c|}
\hline \multirow{3}{*}{$\begin{array}{c}\mathrm{T} \\
\text { (días) }\end{array}$} & \multicolumn{15}{|c|}{ Sitios } \\
\hline & \multicolumn{5}{|c|}{ JS } & \multicolumn{5}{|c|}{ JNS } & \multicolumn{5}{|c|}{$\mathrm{RS}$} \\
\hline & $\begin{array}{l}\mathrm{P} \\
(a)\end{array}$ & $\begin{array}{l}\mathrm{N} \\
(a)\end{array}$ & $\begin{array}{l}\mathrm{Ca} \\
(a)\end{array}$ & $\begin{array}{l}\mathrm{Mg} \\
(a)\end{array}$ & $\begin{array}{l}\mathrm{K} \\
(a)\end{array}$ & $\begin{array}{l}\mathrm{P} \\
\text { (b) }\end{array}$ & $\begin{array}{l}\mathrm{N} \\
(a)\end{array}$ & $\begin{array}{l}\mathrm{Ca} \\
(a)\end{array}$ & $\begin{array}{l}\mathrm{Mg} \\
(a)\end{array}$ & $\begin{array}{c}\mathrm{K} \\
(b)\end{array}$ & $\begin{array}{c}\mathrm{P} \\
(b)\end{array}$ & $\begin{array}{l}\mathrm{N} \\
(a)\end{array}$ & $\begin{array}{l}\mathrm{Ca} \\
(a)\end{array}$ & $\begin{array}{l}\mathrm{Mg} \\
(a)\end{array}$ & $\begin{array}{l}\mathrm{K} \\
(a)\end{array}$ \\
\hline 0 & 0.008 & 1.347 & 0.398 & 0.161 & 0.083 & 0.008 & 1.347 & 0.398 & 0.161 & 0.083 & 0.008 & 1.347 & 0.398 & 0.161 & 0.083 \\
\hline 28 & 0.009 & 1.297 & 0.388 & 0.170 & 0.084 & 0.020 & 1.410 & 0.386 & 0.194 & 0.120 & 0.015 & 1.310 & 0.417 & 0.178 & 0.075 \\
\hline 56 & 0.012 & 1.251 & 0.426 & 0.183 & 0.091 & 0.023 & 1.503 & 0.467 & 0.188 & 0.136 & 0.023 & 1.492 & 0.475 & 0.203 & 0.074 \\
\hline 85 & 0.013 & 1.288 & 0.484 & 0.196 & 0.080 & 0.022 & 1.568 & 0.434 & 0.180 & 0.079 & 0.030 & 1.568 & 0.559 & 0.229 & 0.089 \\
\hline 113 & 0.018 & 1.568 & 0.558 & 0.207 & 0.085 & 0.027 & 1.680 & 0.600 & 0.225 & 0.116 & 0.032 & 1.876 & 0.536 & 0.208 & 0.086 \\
\hline 141 & 0.020 & 1.480 & 0.614 & 0.222 & 0.072 & 0.033 & 1.680 & 0.617 & 0.215 & 0.130 & 0.032 & 1.616 & 0.619 & 0.222 & 0.045 \\
\hline 169 & 0.020 & 1.680 & 0.670 & 0.219 & 0.087 & 0.034 & 2.044 & 0.649 & 0.188 & 0.116 & 0.031 & 2.044 & 0.559 & 0.214 & 0.065 \\
\hline
\end{tabular}

Columnas con letras diferentes para un mismo nutriente denotan diferencias significativas entre sitios $(\mathrm{p}<0.05)$. Sitios experimentales: JS: Jardín subsolado, JNS: Jardín No subsolado, RS: Río Rayo subsolado.

Columns of the same nutrient with different letters are statistically different (Tukey test, $\mathrm{p}<0.05$ ). Experimental sites: JS: Jardín site, plowed soil, JNS: Jardín site, non-plowed soil, RS: Río Rayo site, plowed soil.

y el final del estudio (37.14-23.10, 169.04$69.75,67.14-11.02 \%$, respectivamente) con tendencias similares en JNS y RS (Cuadro 4). La concentración de lignina se incrementó al final del ensayo (12.40-20.20\%), con mayores valores en JS y JNS. La mayoría de los parámetros de calidad de la hojarasca se asociaron significativamente con la MSR (Cuadro 5). Los coeficientes de correlación de Pearson mostraron asociación positiva entre la MSR y los parámetros $\mathrm{C} / \mathrm{N}, \mathrm{N} / \mathrm{P}$ y fenoles totales. La precipitación mostró correlación inversa $(\mathrm{p}<0.05)$ con la MSR en todos los sitios. Las cantidades residuales de N/P y fenoles totales disminuyeron abruptamente al comienzo del estudio (día 28), siendo éstas, en el caso de los fenoles, incluso superiores al $70 \%$.

\section{DISCUSIÓN}

Dinámica de la pérdida de peso de la hojarasca foliar: La evolución de la MSR en los tres sitios mostró una primera etapa en la que la tasa de pérdida de peso fue mayor, hasta el día 141 (Fig. 2), punto a partir del cual disminuyó, siendo ésta mayor en JS. Mayores pérdidas de peso en las primeras etapas del proceso de descomposición, han sido asimismo reportadas por Arellano et al. 2004 y GomaTchimbakala \& Bernhard-Reversat 2006. Éstas se producen seguramente, como resultado del desdoblamiento de los compuestos lábiles de la hojarasca. A esta primera etapa siguió una de menor velocidad, quizás ocasionada por la dominancia de materiales recalcitrantes de más difícil descomposición. Al intentar discernir una posible asociación entre el factor precipitación y la pérdida de peso del material en las bolsas, se encontró una correlación inversa entre ambos, siendo más fuerte para los sitios con igual régimen pluviométrico (JS y JNS) (Cuadro 5). Por el contrario, de acuerdo con el ANOVA, esta situación no se verificó para el tratamiento de subsolado previo del suelo. Así, son probablemente otros factores los que influyen en la descomposición de la hojarasca en 

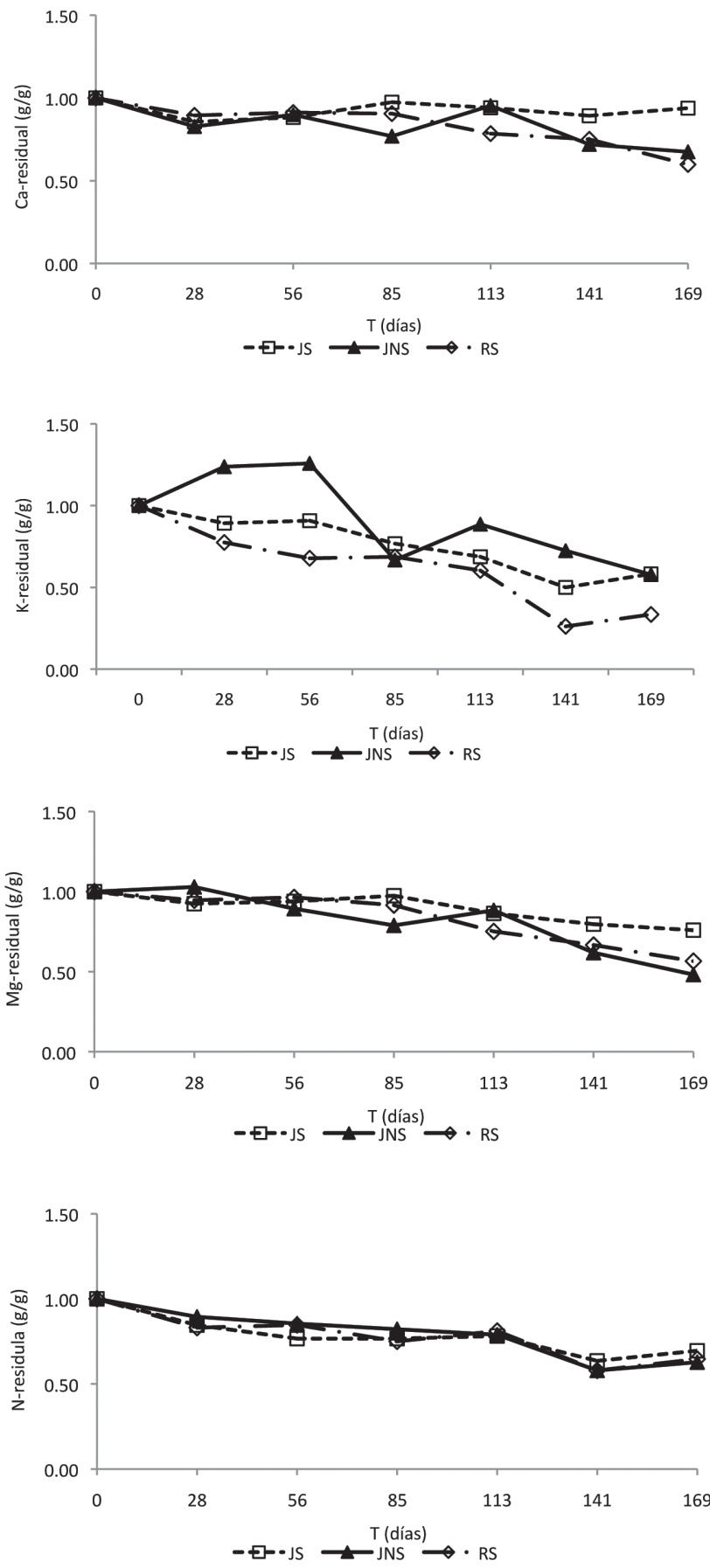

Fig. 3. continua / continued... 


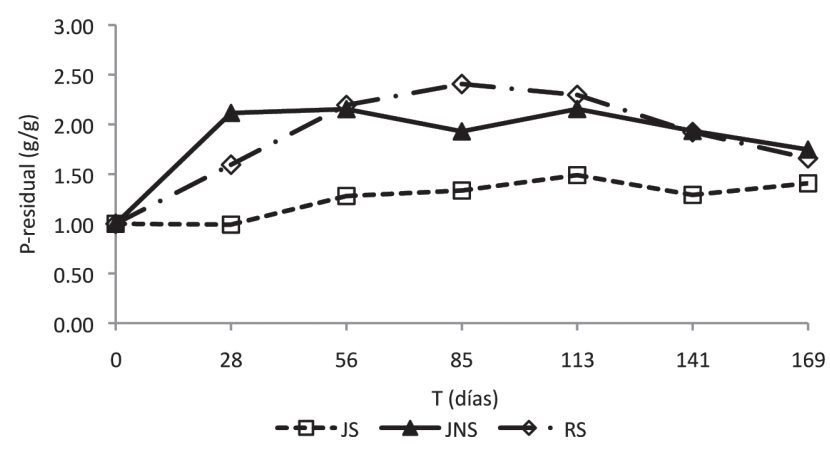

Fig. 3. Cantidades residuales de nutrientes en la hojarasca foliar de A. mangium depositada en las bolsas de descomposición durante el periodo de estudio. Sitios experimentales: JS: Jardín subsolado, JNS: Jardín no subsolado, RS: Río Rayo subsolado.

Fig. 3. Residual elements remaining as a relative quantity of the initial content in the litter bags during the study period. Experimental sites: JS: Jardín site, plowed soil, JNS: Jardín site, non-plowed soil, RS: Río Rayo site, plowed soil.

\section{CUADRO 4}

Calidad de la hojarasca foliar utilizada en el experimento de descomposición en los tres sitios de estudio en las plantaciones de A. mangium del Bajo Cauca, Colombia

TABLE 4

Leaf litter quality employed in the decomposition experiment in three sites located into the A. mangium plantations in the Bajo Cauca region, Colombia

\begin{tabular}{|c|c|c|c|c|c|c|c|c|}
\hline \multirow[b]{2}{*}{ SITIOS } & \multicolumn{8}{|c|}{ Valores iniciales } \\
\hline & $\mathrm{C}$ & $\begin{array}{l}\mathrm{N} \\
\%\end{array}$ & $\mathrm{P}$ & $\mathrm{C} / \mathrm{N}$ & $\mathrm{N} / \mathrm{P}$ & Lignina $\%$ & Lignina/N & Fenoles Totales* \\
\hline JS, JNS y RS & 50.01 & 1.35 & 0.008 & 37.14 & 169.04 & 12.40 & 9.21 & 67.14 \\
\hline \multirow{2}{*}{ SITIOS } & \multicolumn{8}{|c|}{ Valores finales } \\
\hline & C & $\mathrm{N}$ & $\mathrm{P}$ & $\mathrm{C} / \mathrm{N}$ & $\mathrm{N} / \mathrm{P}$ & Lignina & Lignina/N & Fenoles Totales* \\
\hline JS & 44.51 & 1.68 & 0.02 & 26.49 & 83.17 & 19.40 & 11.55 & 10.17 \\
\hline JNS & 39.60 & 2.04 & 0.03 & 19.37 & 60.51 & 21.30 & 10.42 & 16.63 \\
\hline $\mathrm{RS}$ & 47.92 & 2.04 & 0.03 & 23.44 & 65.56 & 19.9 & 9.73 & 6.26 \\
\hline Valores medios & 44.01 & 1.92 & 0.03 & 23.10 & 69.75 & 20.20 & 10.57 & 11.02 \\
\hline
\end{tabular}

* Expresado como \% equivalente de ácido tánico. Sitios experimentales: JS: Jardín subsolado, JNS: Jardín no subsolado, RS: Río Rayo subsolado.

Expressed as \% of tanic acid equivalent. Experimental sites: JS: Jardín site, plowed soil, JNS: Jardín site, non-plowed soil, RS: Río Rayo site, plowed soil. 
CUADRO 5

Coeficientes de correlación de Pearson entre la MSR, la precipitación, y parámetros de la calidad del sustrato en los tres sitios de estudio en las plantaciones de A. mangium del Bajo Cauca, Colombia

TABLE 5

Pearson correlation coefficients computed between residual dry matter (MSR) and precipitation, and quality parameters of the substrate in the three sites of study in the A. mangium plantations in the Bajo Cauca, Colombia

\begin{tabular}{lccc}
\multicolumn{1}{c}{ Parámetros } & JS & JNS & RS \\
$\mathrm{N}$ & -0.76 & -0.87 & -0.83 \\
$\mathrm{P}$ & -0.94 & -0.93 & -0.93 \\
$\mathrm{C} / \mathrm{N}$ & 0.81 & 0.91 & 0.89 \\
$\mathrm{~N} / \mathrm{P}$ & 0.86 & 0.72 & 0.78 \\
Lignina & -0.74 & -0.89 & -0.78 \\
Lignina/N & $-0.24^{*}$ & $-0.38^{*}$ & $0.04 *$ \\
Fenoles totales & 0.71 & 0.64 & 0.74 \\
Precipitación total & -0.70 & -0.75 & -0.46
\end{tabular}

*No significativo $(\mathrm{p}>0.05)$. Sitios experimentales: JS: Jardín subsolado, JNS: Jardín no subsolado, RS: Río Rayo subsolado.

*Non-significant $(\mathrm{p}>0.05)$. Experimental sites: JS: Jardín site, plowed soil, JNS: Jardín site, non-plowed soil, RS: Río Rayo site, plowed soil.

estos sitios, dentro de los cuales han sido propuestos, en su orden: el clima, la composición química de la hojarasca, y la actividad de los microorganismos del suelo (Swift et al. 1979, Aerts \& Chapin 2000). De estos, la calidad de la hojarasca ha sido considerada por numerosos estudios como buena predictora de la descomposición (Xuluc-Tolosa et al. 2003, Ngoran et al. 2006, Martínez-Yerízar et al. 2007, Prause \& Fernández 2007), ya que se han encontrado fuertes relaciones entre la pérdida de peso y algunos indicadores de calidad (v.g. contenido de lignina, fenoles, $\mathrm{N}, \mathrm{C}, \mathrm{P}, \mathrm{C} / \mathrm{N}, \mathrm{C} / \mathrm{P}$, entre otros), lo cual coincide con nuestros hallazgos experimentales (Cuadro 5). La mineralización de un sustrato orgánico generalmente puede ser predicha a partir de su contenido de $\mathrm{N}$ y la relación C/N (Torreta \& Takeda 1999). Así, en los bosques tropicales, cuando la relación $\mathrm{C} / \mathrm{N}$ es menor de $25-40$ y el contenido de $\mathrm{N}$ es mayor que $1.0-2.5 \%$, la mineralización del $\mathrm{N}$ y la descomposición de la materia orgánica son rápidas (Seneviratne et al. 1998, Torreta \& Takeda 1999, Ngoran et al. 2006), particularmente cuando los contenidos de lignina son bajos (Ribeiro et al. 2002). Los valores de estos parámetros de la hojarasca foliar colocada inicialmente en las bolsas, se corresponden con los límites de los intervalos recién indicados, con lo cual sería de esperar una baja tasa de descomposición y una escasa liberación de $\mathrm{N}$ (Bubb et al. 1998); no obstante esta situación no fue aquí observada. Es probable que en ella tengan incidencia los microorganismos del suelo, conforme en estas plantaciones fueron hallados por León et al. (2008): grupos funcionales de bacterias fijadoras de $\mathrm{N}_{2}$ en los nódulos rizobiales; microorganismos solubilizadores de fósforo; y esporas de hongos micorrizo-arbusculares, los cuales cumplen un importante papel en el ciclo biogeoquímico.

Las concentraciones de $\mathrm{N}$ y $\mathrm{P}$ mostraron una fuerte correlación inversa con la MSR (Cuadro 5), lo que denota su influencia individual en la descomposición, aunque necesariamente el proceso se encuentra determinado por un amplio conjunto de variables. En particular se reflejó el carácter limitante del P, ya que la relación N/P se correlacionó positivamente en todos los sitios con la MSR (Cuadro 5), siendo más notorio en JS, donde al final experimento se encontró el máximo absoluto para este parámetro (Cuadro 4), así como la menor pérdida de peso (Fig. 2). La concentración de $\mathrm{P}$ fue similar entre los sitios y mostró incrementos con respecto a su valor inicial (Cuadro 3). Las concentraciones de este nutriente en la hojarasca foliar fueron bajas comparadas con las de otras especies leguminosas tropicales (Palm \& Sánchez 1990), lo que probablemente denota una baja circulación del nutriente a través del sistema suelo-planta, dada su escasa disponibilidad en estos suelos degradados por minería, y la alta movilidad del nutriente en la planta. Los contenidos de lignina del material colocado en las bolsas (Cuadro 4) fueron bajos comparados con los reportados para plantaciones de A. mangium de tierras bajas subtropicales y tropicales 
(Bernhard-Reversat \& Schwartz 1997, Kadir et al. 2001). Aunque el contenido de lignina regula la tasa de descomposición (Bernhard-Reversat 1993), aquí no fue un buen predictor. La correlación negativa de la lignina con la MSR (Cuadro 5), sugiere una mayor descomposición a mayor contenido de lignina, lo cual contrasta con el carácter recalcitrante de este compuesto, que dificulta la degradabilidad de la materia orgánica, y suele reflejarse en menores tasas de descomposición (Meentemeyer 1978, Arunachalam \& Singh 2002, Prause \& Fernández 2007). Al final del experimento, se encontraron incluso incrementos absolutos de su concentración con respecto a los valores iniciales en los tres sitios (Cuadro 4), lo cual señala el aumento de formas recalcitrantes, no obstante, esto no afectó la pérdida de peso del material colocado en las bolsas, ya que la MSR siguió disminuyendo a una tasa relativamente rápida (Fig. 2). Es posible que en esto incida la intervención eficiente de organismos degradadores de este compuesto, como termitas o algunas bacterias $\mathrm{u}$ hongos conforme ha sido reportado por Bernhard-Reversat \& Schwartz 1997, Sariyildiz 2003 y Fioretto et al. 2005. Palm \& Sanchez (1990) señalaron que la concentración de fenoles solubles está más relacionada con las tasas de descomposición, al observar en varias especies de leguminosas, que bajos contenidos de fenoles producían velocidades de descomposición dos veces mayores que las de especies con altos contenidos. El contenido de fenoles aquí determinado fue alto (Cuadro 4) en comparación con los de otros estudios realizados con A. mangium y otras leguminosas tropicales (Kadir et al. 2001, Palm \& Sanchez 1990), y su asociación con la descomposición fue significativa (Cuadro 5).

Velocidad de descomposición de la hojarasca foliar: Las tasas de descomposición aquí obtenidas a través del modelo simple exponencial (Cuadro 2), corresponden con valores intermedios del intervalo reportado para bosques y plantaciones de tierras bajas tropicales $(k=0.1-4.8$ : Sundarapandian \& Swamy 1999, Singh et al. 1999, Kurzatkowski et al.
2004). Esto tiene importantes repercusiones desde la perspectiva de recuperación de áreas degradadas, ya que las tasas de descomposición aquí determinadas suponen el aporte de grandes cantidades de compuestos orgánicos y nutrientes liberados desde la hojarasca, que contribuyen con el restablecimiento de las propiedades físicas y químicas de los suelos y su actividad biológica. Así, cuando se comparan los contenidos en el suelo de materia orgánica, $\mathrm{N}$ y $\mathrm{P}$ entre las plantaciones de A. mangium y los sitios no plantados (Cuadro 1), se encuentra que en los primeros tales valores son entre 2 y 3 órdenes de magnitud superiores. Nuestros valores $k$, obtenidos sobre estos suelos degradados, fueron incluso superiores a los registrados por Berrouet et al. (2003) en bosques húmedos de Colombia $(k=0.54)$ y por McGrath et al. (2000) en plantaciones de Theobroma grandiflorum en la Amazonia Brasilera $(k=0.4)$. La vida media $\left(\mathrm{t}_{0.5}\right)$ aquí determinada fue similar a la reportada por Singh et al. (1999) para Dalbergia sissoo en la India $\left(\mathrm{t}_{0.5}=0.41\right.$ años $)$. El tiempo necesario para que desaparezca el $99 \%$ de la hojarasca $\left(\mathrm{t}_{0.99}\right)$ estuvo alrededor de 2.603.70 años, valores similares a los encontrados por del Valle (2003) en bosques inundables del Pacífico Colombiano, considerados como intermedios para bosques tropicales. La velocidad de descomposición $(k)$ de la hojarasca foliar entre sitios siguió la secuencia decreciente $\mathrm{RS}>\mathrm{JNS}>\mathrm{JS}$, con tendencias similares entre JNS y RS (Cuadro 2). Es posible que las diferencias encontradas sean atribuibles a las condiciones particulares de cada sitio como el microclima, las propiedades edáficas y fundamentalmente la actividad de la biota del suelo (Swift et al. 1979, Attiwill \& Adams 1993, Aerts \& Chapin 2000).

De acuerdo con el modelo doble exponencial, para el sitio JNS ocurrió una rápida degradación de los elementos recalcitrantes (valores $k_{2}$ ). Por otra parte, en los sitios JS y RS se verificó un bajo efecto de $k_{1}$ sobre la descomposición. Estos resultados difieren de los de otros estudios (Berrouet et al. 2003), con valores $k_{1}$ superiores, que indican una más rápida degradación de las fracciones lábiles. 
Estas diferencias en las tasas de descomposición afectan a su vez la velocidad con que los nutrientes de la hojarasca vuelven a estar disponibles para la vegetación. De la velocidad y de la eficiencia con que la planta vuelva a capturarlos, dependerá que estos nutrientes puedan perderse por lavado o lixiviación, o que sean temporalmente fijados en otros componentes del ecosistema como la biota del suelo (Schlesinger 2000, Ribeiro et al. 2002).

Dinámica de nutrientes durante la descomposición de la hojarasca foliar: $\mathrm{La}$ concentración de nutrientes en la MSR (Cuadro 3), mostró una secuencia decreciente: $\mathrm{N}>\mathrm{Ca}>\mathrm{Mg}>\mathrm{K}>\mathrm{P}$, la cual coincide en el caso de $\mathrm{N}$ y $\mathrm{P}$, con la reportada para plantaciones de $A$. mangium (Ngoran et al. 2006). La tendencia de las concentraciones a lo largo del experimento fue el incremento, conforme ha sido encontrado en otros estudios (Singh et al. 2004). Entre otros factores, esta situación puede presentarse como resultado de las deposiciones atmosféricas, posteriormente lavadas del dosel y que alcanzan el piso de las plantaciones; el lavado foliar; la invasión del material por micelio de hongos; y la presencia abundante de microorganismos en el mantillo.

El patrón general de liberación de nutrientes fue: $\mathrm{K}>\mathrm{N}>\mathrm{Mg}>\mathrm{Ca}>\mathrm{P}$ (Fig. 3). La rápida liberación del $\mathrm{K}$ ha sido ampliamente reportada dado su carácter móvil como resultado de no estar ocluido a estructuras orgánicas en los tejidos foliares, sino en forma libre, con lo cual es fácilmente lavado y/o removido (Tukey 1970, Parker 1983). Así, Villela \& Proctor (2002) en bosques tropicales de Pará (Brasil) encontraron pérdidas del $70 \%$ en hojas de Ecclinusa guianensis. Tendencias similares fueron reportadas por Ngoran et al. (2006) para A. mangium, con pérdidas de $\mathrm{K}$ superiores al $80 \%$ al final de su experimento. Aunque no se presentaron diferencias significativas en la liberación de $\mathrm{N}$ entre sitios $(\mathrm{p}=0.97)$, las tendencias fueron similares entre JNS y RS (Fig. 3). Para $\mathrm{N}$ se diferenciaron tres fases de liberación, una lenta inicial (día 113), luego una rápida (día 141) y una final dominada por el proceso de inmovilización (Fig. 3). Este patrón de liberación de $\mathrm{N}$ difiere del de otros estudios (Bubb et al. 1998) y sugiere que éste se encuentra regulado por las condiciones particulares de cada sitio, fundamentalmente por la actividad microbiana y la calidad de la hojarasca (Gallardo-Lancho 2000). La lenta liberación de $\mathrm{N}$ posiblemente se deba a la baja demanda microbiana en el suelo, a pesar de que los incrementos de sus cantidades residuales en el último evento de muestreo, sugieren un proceso de inmovilización, probablemente por el aumento de la biomasa microbiana de organismos colonizadores (Schlesinger 1991).

Las cantidades residuales de $\mathrm{P}$ mostraron tendencias similares entre JNS y RS, evidenciándose un enriquecimiento mayor que el de JS, superando en más del $70 \%$ los valores iniciales al final del ensayo. El patrón de enriquecimiento aquí encontrado para $\mathrm{P}$ difiere del reportado por Ngoran et al. (2006), quienes registraron disminuciones del $\mathrm{P}$ residual en los primeros 180 días del estudio. Aerts (1997) propuso el valor 11.9 como crítico en la hojarasca foliar para la relación N/P. En los bosques tropicales, éste representa algún grado de escasez de $\mathrm{P}$ para los organismos descomponedores, ya que en las células de hongos y bacterias tal relación se encuentra alrededor de 10-15. El valor N/P inicial en este estudio (169: Cuadro 5) denota un claro déficit de P, confirmando nuestros hallazgos experimentales con respecto a la inmovilización biológica del nutriente, y/o la ocurrencia de un proceso de retranslocación del $\mathrm{P}$, conforme ha sido hallado en estas plantaciones (Castellanos-Barliza \& León, en prep.). En el caso de $\mathrm{Ca}$, su lenta liberación, especialmente clara en JS, podría ser debida, entre otros factores, a una inmovilización biológica, que podría estar acentuada en estos suelos ácidos con altas concentraciones de Fe (Duchaufour 1984, Martin et al. 1996). Estos resultados contrastan con los de Liao et al. (2006) en bosques húmedos de Taiwan, en donde el Ca mostró la mayor movilidad durante el proceso de descomposición, superando las tendencias generales de liberación que muestran $\mathrm{N}$ y $\mathrm{P}$ en los bosques tropicales. La menor liberación de Mg se presentó en JS, 
donde al final del estudio solo se había liberado $20 \%$ de su cantidad inicial, no obstante, al igual que para $\mathrm{Ca}$, no se encontraron diferencias significativas entre sitios $(\mathrm{p}=0.61)$. Los patrones de liberación de $\mathrm{Ca}$ y $\mathrm{Mg}$, podrían estar muy seguramente afectados por los incrementos en las concentraciones foliares conforme avanza el proceso de descomposición (Cuadro 3), los cuales fueron mayores en JS. Estos incrementos han sido usualmente atribuidos a la contaminación del material confinado en las bolsas por suelo mineral, así como por los pluviolavados (Ewel 1976).

En síntesis, la descomposición de la hojarasca foliar de A. mangium se caracterizó por ser relativamente rápida, aun cuando las tasas se determinaron sobre suelos degradados por minería aurífera. La práctica del subsolado previo establecimiento de las plantaciones no tuvo un efecto determinante sobre la descomposición. Por el contrario, algunas variables de calidad del sustrato y la precipitación, se presentaron como buenas predictoras del proceso.

Los aportes de materia orgánica y de nutrientes derivados de la descomposición de la hojarasca fina en estas plantaciones, ha conllevado el mejoramiento sustancial de estos suelos degradados, conforme se deriva de incrementos medios cercanos al $300 \%$ en materia orgánica, $200 \%$ en N y $340 \%$ en P, al ser comparadas con sitios no plantados con la especie. En general, se registró una liberación efectiva de elementos como $\mathrm{Ca}, \mathrm{Mg}$ y $\mathrm{K}$, con patrones similares entre ellos. El P en particular, fue el elemento más restrictivo para la descomposición y mineralización de la materia orgánica, mostrando tendencia a la inmovilización, dada su escasa disponibilidad edáfica. Los resultados aquí obtenidos permiten señalar que la especie presenta un gran potencial para la recuperación de sitios afectados por diferentes procesos degradativos, especialmente aquellos que dejan el suelo con bajos o nulos contenidos de materia orgánica y nutrientes, que impiden un adecuado proceso de colonización vegetal.

\section{AGRADECIMIENTOS}

A la Corporación Autónoma Regional del Centro de Antioquia (CORANTIOQUIA) por la financiación del proyecto "Evaluación silvicultural de las plantaciones de Acacia mangium Willd. y su papel en la recuperación de suelos en el Bajo Cauca Antioqueño" (Contrato 7214 del 2006). A Nolberto Marín, Luis Fernando Osorio, Juan Carlos Guingue, Miguel Guzmán, Gloria Mazo y Benjamín Rojano por el seguimiento y acompañamiento técnico durante la ejecución del proyecto. Al Área de Biogeoquímica del Laboratorio de Ecología Cesar Pérez Figueroa y al Laboratorio de Alimentos de la Universidad Nacional de Colombia, Sede Medellín, por sus aportes en el procesamiento y determinación analítica de muestras. A Diego Navarrete, Walter Osorio y Álvaro Lema por sus valiosas anotaciones para la elaboración de este documento.

\section{RESUMEN}

La descomposición de hojarasca en los ecosistemas terrestres está regulada por varios factores, entre ellos, la humedad, temperatura, calidad de la hojarasca y la actividad de los microoganismos. Estudiamos el efecto de la precipitación y el tratamiento de subsolado del suelo previo establecimiento de plantaciones de Acacia mangium, usando la técnica de bolsas de descomposición durante seis meses en plantaciones del Bajo Cauca (Colombia). La constante de descomposición anual ( $k$ ) del modelo simple exponencial, osciló entre 1.24 y 1.80. Las constantes $k_{1}$ y $k_{2}$ del modelo doble exponencial fluctuaron entre 0.88-1.81 y 0.58-7.01. Al final del experimento la materia seca residual promedio (MSR) fue del 47\%. Las cantidades residuales de $\mathrm{N}$, Ca y $\mathrm{Mg}$ en la hojarasca de A. mangium mostraron un patrón de liberación lento. La evolución del P en la MSR mostró un proceso dominante de inmovilización, dada su baja disponibilidad en el suelo. Algunos parámetros de calidad del sustrato (N, P, C/N, N/P y fenoles) fueron buenos predictores del proceso, así como la precipitación, situación contraria a la del tratamiento de subsolado.

Palabras clave: Acacia mangium, subsolado, bolsas de descomposición, liberación de nutrientes, tasas de descomposición, recuperación de suelos. 


\section{REFERENCIAS}

Aerts, R. 1997. Climate, leaf litter chemistry and leaf litter decomposition in terrestrial ecosystems: a triangular relationship. Oikos 79: 439-449.

Aerts, R. \& F.S. Chapin. 2000. The mineral nutrition of wild plants revisited: a re-evaluation of processes and patterns. Adv. Ecol. Res. 30: 1-67.

Arellano, R., J. Paolini, L. Vásquez \& E. Mora. 2004. Producción y descomposición de hojarasca en tres agroecosistemas de café en el estado de Trujillo, Venezuela. Rev. Forest. Venez. 48: 7-14.

Arunachalam, A. \& N.D. Singh. 2002. Leaf litter decomposition of evergreen and deciduous Dillenia species in humid tropics of north-east India. J. Trop. Forest. Sci. 14: 105-115.

Attiwill, P.M. \& M.A. Adams. 1993. Tansley Review No 50. Nutrient cycling in forests. New Phytol. 124: $561-582$

Bernhard-Reversat, F. 1993. Dynamics of litter and organic matter at the soil-litter interface in fast-growing tree plantations on sandy ferrallitic soils (Congo). Acta Oecol. 14: 179-195

Bernhard-Reversat, F. \& D. Schwartz. 1997. Change in lignin content during litter decomposition in tropical forest soils (Congo): Comparison of exotic plantations and native stands. Earth. Planet. Sci. Lett. 325: 427-432.

Berrouet, L.M., L.M. Loaiza \& F. Moreno. 2003. Descomposición de la hojarasca fina en bosques de la cuenca media del río Porce, p. 36-43. In M.C. Díez \& J.D. León (eds.). Primer Simposio Forestal. Universidad Nacional de Colombia, Facultad de Ciencias Agropecuarias, Medellín.

Bubb, K.A., Z.H. Xu, J.A. Simpson \& P.G. Safigna. 1998. Some nutrient dynamics associated with litterfall and litter decomposition in hoop pine plantations of southeast Queensland, Australia. Forest Ecol. Manag. 110: 343-352.

Bunnell, F. \& D.E.N. Tait. 1974. Mathematical simulation models of decomposition soil organisms and decomposition in tundra, p. 207-225. In A.J. Holding, S.F Heal, Jr. Maclean \& P.W. Flanagan (eds.). Soil organisms and decomposition in tundra. Tundra Biome Steering Committee, Estocolmo.

Del Valle, J.I. 2003. Descomposición de la hojarasca fina en bosques pantanosos del Pacífico Colombiano. Interciencia 28: 148-153.
Duchafour, P.H. 1984. Edafología I. Edafogénesis y clasificación. Masson, Barcelona, España.

Duponnois, A \& Bâ A.M. 1999. Growth stimulation of Acacia mangium Willd by Pisolithus sp. in some Senegalese soils. Forest Ecol. Manag. 119: 209-215.

Ewel, J.J. 1976. Litter fall and leaf decomposition in a tropical forest succession in eastern Guatemala. J. Ecol. 64: 293-308.

Fioretto, A., C. Di Nardo, S. Papa \& A. Fuggi. 2005. Lignin and cellulose degradation and nitrogen dynamics during decomposition of three leaf litter species in a Mediterranean ecosystem. Soil Biol. Biochem. 37: 1083-1091.

Gallardo-Lancho, J.F. 2000. Biogeochemistry of Mediterranean forest ecosystems, a case study, p. 423-460. In J.M. Bollag \& G. Stotzky (eds.). Soil Biochemistry 10, Marcel Dekker, Nueva York, EEUU.

Goma-Tchimbakala, J. \& F. Bernhard-Reversat 2006. Comparison of litter dynamics in three plantations of an indigenous timber-tree species (Terminalia superba) and a natural tropical forest in Mayombe, Congo. Forest Ecol. Manag. 229: 304-313.

Horwitz, W. 2003. Official methods of analysis of AOAC International. Association of official analytical chemists. Arlington, Virginia, EEUU.

Huang, J., X. Wang \& E. Yan. 2007. Leaf nutrient concentration, nutrient resorption and litter decomposition in an evergreen broad-leaved forest in eastern China. Forest Ecol. Manag. 239: 150-158.

Intituto Geográfico Agustín Codazzi (IGAC). 2007. Levantamiento semidetallado de las coberturas terrestres. Departamento de Antioquia, Subdirección Agrología, Bogotá, Colombia.

Jordan, C.F. 1985. Nutrient cycling in tropical forest ecosystems. Principles and their application in management and conservation. Wiley, Nuava York, EEUU.

Kadir, W.R., O.V. Cleemput \& A.R. Zaharah. 2001. Microbial respiration and nitrogen release patters of decomposing Acacia mangium leaf litter from Kemasul forest reserve, Malaysa. J. Trop. Forest Sci. 13: 1-12.

Kumar, R. \& M. Agrawal. 2001. Litterfall, litter decomposition and nutrient release in five exotic plant species planted on coal mine spoils. Pedobiologia 45: 298-312.

Kurzatkowski, D., C. Martius, H. Höfer, M. Garcia, B. Förster, L. Beck \& P. Vlek. 2004. Litter decomposition, microbial biomass and activity of soil organisms 
in three agroforestry sites in central Amazonia. Nutr. Cycling Agroecosyst. 69: 257-267.

León, J.D., M.C. Díez, J. Castellanos, L.F. Osorio \& N. Marín. 2008. Grupos funcionales de microorganismos en suelos degradados por minería de aluvión plantados con Acacia mangium. Suelos Ecuatoriales 38: $75-80$.

Liao, J.H., H.H. Wang, CH.CH. Tsai \& Z.Y. Hseu. 2006. Litter production, decomposition and nutrient return of uplifted Coral Reef tropical forest. Forest Ecol. Manag. 235: 4-185.

Lim, M.T. 1988. Studies on Acacia mangium in Kemasul forest, Malaysia. I. Biomass and productivity. J. Trop. Ecol. 4: 293-302.

Martín, A., Gallardo J.F. \& I. Santa Regina. 1996. Aboveground litter production and bioelement potential return in an evergreen oak (Quercus rotundifolia) woodland near Salamanca (Spain). Ann. Sci. Forest. 53: 811-818.

Martínez-Yarízar, A., S. Nuñez \& A. Búrquez. 2007. Leaf litter decomposition in a southern sonoran desert ecosystem, northwestern México: Effects of habitat and litter quality. Acta Oecol. 32: 291-300.

Mcgrath, A.D., N.B. Comerford \& M.L. Duryea. 2000 Litter dynamics and monthly fluctuations in soil phosphorus availability in an Amazonian agroforest. Forest Ecol. Manag. 131: 167-181.

Meentemeyer, V. 1978. Macroclimate the lignin control of litter decomposition rates. Ecology 59: 465-472.

Montagnini, F. \& C.F. Jordan. 2002. Reciclaje de nutrientes, p.167-191. In M.R. Guariguata \& G. Kattan (eds.). Ecología y conservación de bosques neotropicales. Editorial tecnológica, Cartago, Costa Rica.

Ngoran, A., N. Zakra, K. Ballo, C. Kouamé, F. Zapata, G. Hofman \& O. Van Cleemput. 2006. Litter decomposition of Acacia auriculiformis Cunn. Ex Benth. and Acacia mangium Willd. under coconut trees on quaternary sandy soils in Ivory Coast. Biol. Fertil. Soils. 43: 102-106.

Olson, J.S. 1963. Energy storage and balance of producers and decomposer in ecological systems. Ecology 44 322-331.

Osorio, P.I. 2000. Efecto de la Acacia mangium en algunas propiedades físicas y químicas en suelos degradados por minería de aluvión en el Bajo Cauca Antioqueño. Trabajo de grado en la Maestría de Bosques y Conservación Ambiental, Facultad de Ciencias
Agropecuarias, Universidad Nacional de Colombia, Medellín, Colombia.

Palm, C.A. \& P.A. Sanchez. 1990. Decomposition and nutrient release patterns of the leaves of three tropical legumes. Biotropica 22: 330-338.

Palma, R.M., J. Prause, A.V. Fontanive \& M.P. Jimenez.1998. Litter fall and litter decomposition in a forest of the Parque Chaqueño Argentino. Forest Ecol. Manag. 106: 205-210.

Parker, G.G. 1983. Throughfall and stemflow in the forest nutrient cycle. Adv. Ecol. Res. 13: 57-133.

Prause, J. \& C. Fernández. 2007. Litter decomposition and lignin/cellulose and lignin/total nitrogen rates of leaves in four species of the Argentine Subtropical forest. Agrochimica 51: 294-300.

Ribeiro, C., M. Madeira \& M.C. Araújo. 2002. Decomposition and nutrient release from leaf litter of Eucalyptus globulus grown under different water and nutrient regimes Forest Ecol. Manag. 171: 31-41.

Sariyildiz, T. 2003. Litter decomposition of Picea orientalis, Pinus sylvestris and Castanea sativa trees grown in Artvin in relation to their initial litter quality variables. Turk. J. Agr. Forest. 27: 237-243.

Schlesinger, W.H. 1991. Biogeochemistry: An analysis of global change. Academic, Nueva York, EEUU.

Schlesinger, W.H. 2000. Biogeoquímica: un análisis global. Ariel Ciencia, Barcelona, España.

Seneviratne, G., L.H.J. Van Holm \& S.A. Kulasooriya. 1998. Quality of different mulch materials and their decomposition and $\mathrm{N}$ release under low moisture regimes. Biol. Fertil. Soils. 26: 136-140.

Singh, K.P., P.K. Singh \& S.K. Tripathi. 1999. Litterfall, litter decomposition and nutrient release patterns in four native tree species raised on coal mine spoil at Singrauli, India. Biol. Fertil. Soils 29: 371-378.

Singh, R., R. Kumar \& M. Agrawal. 2004. Litter decomposition and nutrient release in relation to atmospheric deposition of $\mathrm{S}$ and $\mathrm{N}$ in a dry tropical region. Pedobiologia 48: 305-311.

Singleton, V.L. \& J.A. Rossi. 1965. Colorimetry of total phenolics with phosphomolybdic-phosphotungstic acid reagents. Am. J. Enol. Vitic. 16: 58-144.

Sundarapandian, S.M. \& P.S. Swamy. 1999. Litter production and leaf-litter decomposition of selected tree species in a tropical forest at Kodayar in the western Ghats, India. Forest Ecol. Manag. 123: 231-244. 
Swift, M.J. \& J.M. Anderson. 1989. Decomposition, p.547569.In H. Lieth \& M.J.A. Werger (eds.). Tropical rain forest ecosystems: Biogeographical and ecological studies. Ecosystems of the world 14A. Elsevier Science, Nueva York, EEUU.

Swift, M.J., O.W. Heal, Anderson, J.M. 1979. Decomposition in terrestrial ecosystems. Oxford, England.

Tejada, I. 1992. Control de calidad y análisis de alimentos para animales. Secretaría de Educación Pública. Dirección General de Derechos de Autor. Número de registro 17222 . México D.F.

Torres, D.A. \& J.I. del Valle. 2007. Growth and yield modelling of Acacia mangium in Colombia. New Forest. 34: 293-305.

Torreta, N.K. \& H. Takeda. 1999. Carbon and nitrogen dynamics of decomposing leaf litter in a tropical hill evergreen forest. Eur. J. Soil Biol. 35: 57-63.

Tsukamoto, J. \& J. Sabang. 2005. Soil macro-fauna in an Acacia mangium plantation in comparison to that in a primary mixed dipterocarp forest in the lowlands of Sarawak, Malaysia. Pedobiologia 49: 69-80.

Tukey, H.B. Jr. 1970. The leaching of substances from plants. Ann. Rev. Plant Physiol. 21: 305-324.

Universidad Nacional de Colombia-Corantioquia. 2008. Evaluación silvicultural de las plantaciones de Acacia mangium Willd. y su papel en la recuperación de suelos en el Bajo Cauca Antioqueño. Informe final Contrato 7214 de 2006. Universidad Nacional de Colombia, Medellín, Colombia.

Villela, D.M. \& J. Proctor. 2002. Leaf litter decomposition and monodominance in the Peltogyne forest of Maracá island, Brazil. Biotropica 34: 334-347.

Wang, Q., S. Wang \& Y. Huang. 2008. Comparisons of litterfall, litter decomposition and nutrient return in a monoculture Cunninghamia lanceolata and a mixed stand in southern China. Forest Ecol. Manag. 255: $1210-1218$

Weerakkody, J. \& D. Parkinson. 2006a. Input, accumulation and turnover of organic matter, nitrogen and phosphorus in surface organic layers of an upper montane rainforest in Sri Lanka. Pedobiologia 50: 377-383.

Weerakkody, J. \& D. Parkinson. 2006b. Leaf litter decomposition in an upper montane rainforest in Sri Lanka. Pedobiologia 50: 387-395.

Wieder, R.K. \& G.E. Lang. 1982. A critique of the analytical methods used in examining decomposition data obtained from litter bags. Ecology 63: 1636-1642.

Xuluc-Tolosa, F.J., H.F.M. Vestera, N. Ramírez-Marcial, J. Castellanos-Albores \& D. Lawrence. 2003. Leaf litter decomposition of tree species in three successional phases of tropical dry secondary forest in Campeche, México. Forest Ecol. Manag. 174: 401-412. 